\title{
Digestibility of feeds in broiler chicken (Galus galus linnaeus, 1758) in Africa: a review
}

\author{
Ghislaine Sègbédji Théodora ATCHADE ${ }^{1 *}$, Frédéric Mankpondji HOUNDONOUGBO ${ }^{2}$, \\ Christophe Archille Armand Mahoussi CHRYSOSTOME ${ }^{2}$ and \\ Guy Apollinaire MENSAH ${ }^{1}$
}

\author{
${ }^{1}$ Laboratoire de Recherches Zootechnique Vétérinaire et Halieutique, Institut National des Recherches \\ Agricoles du Bénin (INRAB). \\ ${ }^{2}$ Laboratoire de Recherche Avicole et de Zoo-Economie, Faculté des Sciences Agronomiques de l'Université \\ d'Abomey-Calavi, Bénin. \\ ${ }^{*}$ Corresponding author; E-mail: atchadedora@yahoo.fr; Tel: + (229) 66002855
}

\begin{abstract}
Feed digestibility indicates how much the body uses feed and its nutrients. This study aimed at making a literature review on the digestibility of balanced feed given to broiler chicken in Africa. Data used for this literature review were collected through many scientific papers published from 2008 to 2018 and uploaded to Google Scholar, Pubmed, Agora search engines and in national university libraries. Numerous scientific studies in this field indicate that more than forty feedstuffs are commonly used to formulate balanced feed delivered to broiler chicken in modern system in Africa. Feed digestibility is generally assessed in vivo by the direct method in broiler chicken. The data compiled shows that feed digestibility of broiler chicken varies enormously depending on the feedstuffs in balanced feed and also on their incorporation rate. This literature review reveals that balanced feeds used to feed broiler has Apparent Digestibility more than $50 \%$.
\end{abstract}

(C) 2019 International Formulae Group. All rights reserved

Keywords: Balanced feed, In vivo digestibility, Broiler chicken, Africa.

\section{INTRODUCTION}

In modern poultry production, breeders produce themselves or purchase feeds made by products and byproducts, minerals and vitamins (Traore and Bebay, 2006). Thus, balanced feed offered to broiler may vary depending on the available feedstuffs. However, this balanced feed can be digested differently by broiler chicken. It must undergo a series of physical and chemical actions prior to obtaining simple, absorbable constituents called nutrients (Sauvant, 2004). In broiler, the transit of food for their digestion is relatively fast and lasts an average of twentyfour (24) hours (Deiltel et al., 2012).

Ingested feed are not absorbed in total by body: part of the ingestas is not used and passes through the digestive tract and is found excreted in faeces (Deiltel et al., 2012). "Feed digestibility" indicates how much the body uses feeds or nutrients (Deiltel et al., 2012). Nutrient digestibility refers to the notion of bioavailability. It depends on the animal (species, strain, age), the composition of feedstuff, and eventual technological treatments (thermal and / or mechanical). 
A digestible fraction of food is the part of the "ingesta" that is not found in the faeces. Digestibility is the main factor of variation in the energy value of feed. Digestibility is therefore an index of the disappearance of food in the intestine, but not of the efficiency of use of food by animal metabolism. A food can be very digestible without providing enough nutrients to make up needs of animal.

\section{FEED DIGESTIBILITY IN BROILER AND BALANCED EXPERIMENT}

\section{Feed digestibility in broiler chicken}

The feed ingested by chicken is not entirely used by his body; part of "ingestats" passes through the digestive tract and is found in the faeces. In poultry, the determination of digestibility is complex and very delicate, because the faeces and urine are evacuated together by the same orifice which is the cloaca (Dimi, 1990). Urine compounds are mostly nitrogenous. The separation is then based on the fact that most of the urinary nitrogen is in the form of uric acid and that almost all of the fecal nitrogen is of a protein nature (Mahoungou-Mouéllé, 1996). Consequently the requirement and feed composition are expressed in metabolizable energy.

In vivo digestibility study makes it possible to better understand the percentage of nutrient utilization (organic matter, amino acids, fat and metabolizable energy) absorbed in digestive tract and which constitutes the fraction that can be used for metabolism (INRA, 1989). It is a quantitative concept that results in digestibility coefficient (INRA, 1989, Adoum, 2007). There are two types of digestibility coefficients: Apparent Digestibility Coefficient and the Real Digestibility Coefficient.

Most studies on broiler chicken in Africa focus on feed apparent digestibility, limiting themselves to the balance between the nutrients of ingestats and excretas.

\section{Determination of Apparent Digestibility in broiler chicken}

According to Rivière (1991), food digestibility can be determined from three
(03) groups of methods: (i) In vivo methods; (ii) in vitro methods (laboratory method); iii) Mathematical or prediction methods.

Direct method in vivo is used in the scientific papers used for this literature review. It is based on faecal digestibility measurements, the method of which was standardized in 1995 by the EGRAN group (Guermah, 2012). Representative sampling is used because slight differences in digestive function in broiler of the same breed, age and sex. Repetitions allow the detection of errors (Rivière, 1991). Feed samples as well as faeces samples are dried and used in the laboratory to determine their chemical composition according to the methods recommended by AOAC (1991); AOAC (2000). This chemical composition includes nutrients such as Dry Matter (DM), Crude Protein (CP), Fatty Matter (EE), and Crude Fiber (CF).

A typical digestibility experiment is conducted over a period of five (05) to ten (10) days by feeding daily experimental chicken. Before starting digestibility test, a period of acclimatization and food transition of 3 to 4 days is observed. To the experimental period are added stages of droppings drying, grinding and analysis (Bourdillon et al., 1990). Experiment on the assessment of feed digestibility in broiler chicken is carried out in digestibility cages equipped with a faeces collection device, on chickens that age and sex are known. Broilers are individually arranged per digestibility cage and data is collected daily by chicken. Thus, the quantities of feed delivered and refused as well as those of faeces are weighed and recorded daily.

After evaluation of the chemical composition of samples of feed served, refused and faeces, Apparent Digestibility of the experimental diet in its nutrients is calculated by its following formula:

Apparent digestibility $=[(\%$ Nutrients in feed X FI) - (\%Nutrients in faeces X FO)]/ $\%$ Nutrients in feed X FI x100;

Where $\mathrm{FI}=$ feed intake and $\mathrm{FO}=$ faecal output Apparent digestibility determination in broiler chicken. 


\section{CONCENTRATED FEEDSTUFFS IN BROILER CHICKEN BALANCED FEED IN AFRICA}

The rich tropical biodiversity, the great diversity of agricultural production systems in African countries in general and the presence of small agro-processing units contribute to a wide range of feedstuffs that can be used to feed animals (Archimède et al., 2011). The diet of chicken and rabbit generally involves two main types of concentrated feedstuffs (CFS): cereals and by-products.

These CFS include crop products; agro-industrial food and animal products and by-products. Their use depends mainly on the availability in the region, the season, the price and their ability to be kept for a long time (Toléba et al., 2007). Forty-four (44) different CFS used in the feeding of chicken in Africa and listed in this literature review are: Baobab leaf meal, Blood meal, Brewer Dried grains, Cassava flour, Cassava peel meal, Cottonseed cake, Dacryodes edulis Seed Meal, Degraded brewer dried grains, Ethanol-treated castor oil seed meal, Fermented Parkia biglobosa seed meal, Fermented Terminalia catappa whole fruit meal, Fish meal, Fresh bovine rumen epithelia tissues scrapings, Full fat soybean meal, Groundnut meal, Lime juice, Local processed groundnut cake meal, Maize (Yellow), Maize bran, Maize offal, Maize, Moringa leaf meal, Oil-sunflower, Okara, Palm kermel cake, Palm kernel meal, Palm oil, Palm oil sludge, Rye, Senna obtusifolia seed meal (raw, boiled, soaked, fermented), Sorghum varieties, Soy oil, Soya bean meal, Soya beans, Sunflower meal, Sweet orange peel meal, Treated Banana Peel Meal, Undecorticated Sunflower Seed Meal, Undegraded brewer dried grains, Urea fermented brewer's dried grains, Vegetable oil blend, Wheal Offal, Wheat bran, Whole soyabean.

Moreover, in the modern system, the diet of the broiler chicken is also based on the use of some vitamins, minerals amino acids and pigments (N'gom, 2004; Guindjoumbi,
2007; Kpodékon et al., 2009). In this category, twenty-two (22) feedstuffs in this category are commonly used in Africa. These feedstuffs are: Bone meal, Celite, Choline chloride, Chromic oxide premix, DCP, Dextrose, Dicalcium Phosphate, DL Methionine, Lime stone, L-Lysine, L-Lysine-HCl, Methionine, Mineral premix, Monocalcium phosphate, Oyster shell, Premix, Salt, Sodium bicarbonate, Sodium Chloride, Vitamin, Vitamin/mineral premix, Washed building sand.

\begin{tabular}{lllr} 
APPARENT & \multicolumn{2}{c}{ DIGESTIBILITY } & OF \\
BALANCED & FEED IN & BROILER \\
CHICKEN & & &
\end{tabular}

Several authors in Africa studied feed digestibility in improved chicken fed with balanced diets based on various conventional and unconventional concentrated feedstuffs. Table 1 presents the digestibility of balanced feeds in broiler chicken in Africa. The apparent digestibility varied according rate of the feedstuff tested in study. Indeed, most of these digestibilities have been evaluated following the use of a feedstuff at different levels of incorporation into a basic diet formula. Majority of nutrients in balanced feeds delivered to broiler chicken in Africa are well digested. Digestibility Coefficient of the dry matter in balanced feed varied greatly from a study to another: they are greater than $55 \%$ and range from 56 to $97 \%$. The lowest Digestibility Coefficient of dry matter is $56 \%$. The digestibility of crude protein varies from 52 to $92 \%$. However, a very low crude protein digestibility ranging from 13 to $47 \%$ were recorded in studies. The lowest fat digestibility recorded was $6 \%$ when most of fat digestibility varies from 46 to $93 \%$. Crude fiber whose high content in balanced feed hampers the proper functioning of the digestive system are digested at a rate ranging from 50 to $76 \%$. However, some low digestibilities of crude fiber ranging from 6 to $46 \%$ are recorded. 
Tableau 1: Apparent digestibility of some balanced feeds in broiler in Africa.

\begin{tabular}{|c|c|c|c|c|c|c|}
\hline $\begin{array}{l}\text { Experimental Feedstuffs } \\
\text { (Rate in the feed) }\end{array}$ & Feed composition & $\begin{array}{c}\text { DDM } \\
(\%)\end{array}$ & $\begin{array}{l}\text { DCP } \\
(\%)\end{array}$ & $\begin{array}{l}\text { DEE } \\
(\%)\end{array}$ & $\begin{array}{l}\text { DCF } \\
(\%)\end{array}$ & $\begin{array}{l}\text { Authors } \\
\text { (Country) }\end{array}$ \\
\hline $\begin{array}{l}\text { Ethanol-treated castor oil } \\
\text { seed meal }(10 ; 15 ; 20 \\
\mathrm{kg} / 100 \mathrm{~kg})\end{array}$ & $\begin{array}{l}\text { Ethanol-treated castor } \\
\text { oil seed meal, Maize, } \\
\text { Soya bean meal, Fish } \\
\text { meal, Wheat offal, } \\
\text { Bone meal, Oyster } \\
\text { shell, Lysine, } \\
\text { Methionine, Vit.Min } \\
\text { premix, Salt }\end{array}$ & $\begin{array}{l}84.49- \\
84.91\end{array}$ & $\begin{array}{l}67.59- \\
73.99\end{array}$ & $\begin{array}{l}74.53- \\
76.98\end{array}$ & $\begin{array}{l}60.02- \\
63.65\end{array}$ & $\begin{array}{l}\text { Sobayo et al., } \\
2012 \\
\text { (Nigeria) }\end{array}$ \\
\hline $\begin{array}{l}\text { Acid organic }(0.25 \mathrm{~kg} / 100 \\
\mathrm{kg})\end{array}$ & $\begin{array}{l}\text { Acid organic, Maize, } \\
\text { Soybean meal, Fish } \\
\text { meal, Palm kermel } \\
\text { cake; Bone meal, Salt, } \\
\text { Lysine, Methionine, } \\
\text { Premix }\end{array}$ & 66.44 & 62.87 & 60.76 & 31.82 & $\begin{array}{c}\text { Ndelekwete et } \\
\text { al., } 2016 \\
\text { (Nigeria) }\end{array}$ \\
\hline $\begin{array}{l}\text { Fermented Parkia } \\
\text { biglobosa seed meal }(0 ; \\
5.75 ; 11.50 ; 17.25 ; 23 \\
\mathrm{~kg} / 100 \mathrm{~kg})\end{array}$ & $\begin{array}{l}\text { Fermented Parkia } \\
\text { biglobosa seed meal, } \\
\text { Maize, Wheat offal, } \\
\text { Groundnut cake, Palm } \\
\text { kernel cake, Fish meal, } \\
\text { Blood meal, Bone } \\
\text { meal, Lysine, } \\
\text { Methionine, } \\
\text { Vit/premix, Salt }\end{array}$ & ND & $\begin{array}{l}72.34- \\
89.15\end{array}$ & $\begin{array}{c}81.71- \\
92\end{array}$ & $\begin{array}{l}51.74- \\
68.37\end{array}$ & $\begin{array}{l}\text { Obun, } 2008 \\
\text { (Nigeria) }\end{array}$ \\
\hline $\begin{array}{l}\text { Moringa leaf meal, } \\
\text { Baobab leaf meal }(0 ; 0.15 \\
\mathrm{kg} / 100 \mathrm{~kg})\end{array}$ & $\begin{array}{l}\text { Moringa leaf meal, } \\
\text { Baobab leaf meal, } \\
\text { Maize, Soyabean, } \\
\text { Wheat offal, Fish } \\
\text { meal, Bone meal, } \\
\text { Premix, Methionine, } \\
\text { Lysine, Salt }\end{array}$ & $\begin{array}{l}66.26- \\
79.66\end{array}$ & $\begin{array}{l}81.71- \\
88.27\end{array}$ & $\begin{array}{l}66.46- \\
83.24\end{array}$ & ND & $\begin{array}{l}\text { Igwebuike et } \\
\text { al., } 2017 \\
\text { (Nigéria) }\end{array}$ \\
\hline $\begin{array}{l}\text { Avizyme } 1505, \text { Phyzyme } \\
\text { xp }(0 ; 0.07 ; 0.2 \mathrm{~g} / 1000 \mathrm{~g})\end{array}$ & $\begin{array}{l}\text { Avizyme } 1505, \\
\text { Phyzyme xp, Maize, } \\
\text { Soybean meal, Full fat } \\
\text { soybean meal, Rye, } \\
\text { Vegetable oil blend, } \\
\text { Dicalcium Phosphate, } \\
\text { Limestone, Nacl, } \\
\text { Vit+Premix, DL- } \\
\text { Methionine, L-Lysine } \\
\text { Washed builiding sand }\end{array}$ & $\begin{array}{l}74.3- \\
75.7\end{array}$ & $\begin{array}{l}65.9- \\
71.4\end{array}$ & $\begin{array}{l}77.9- \\
84.1\end{array}$ & $\begin{array}{l}13.4- \\
16.3\end{array}$ & $\begin{array}{c}\text { Youssef et al., } \\
2014 \\
\text { (Egypt) }\end{array}$ \\
\hline $\begin{array}{l}\text { African yam bean cake }(0 \\
3.86 ; 7.72 ; 11.65 ; 15.50\end{array}$ & $\begin{array}{l}\text { African yam bean } \\
\text { cake, Maize, Soybean, }\end{array}$ & $\begin{array}{l}96.45- \\
96.97\end{array}$ & $\begin{array}{l}38.86- \\
40.42\end{array}$ & $\begin{array}{l}52.02- \\
52.97\end{array}$ & $\begin{array}{c}63.72- \\
65.11\end{array}$ & $\begin{array}{l}\text { Raji et al., } 2015 \\
\text { (Nigéria) }\end{array}$ \\
\hline
\end{tabular}




\begin{tabular}{|c|c|c|c|c|c|c|}
\hline $\begin{array}{l}\text { Experimental Feedstuffs } \\
\text { (Rate in the feed) }\end{array}$ & Feed composition & $\begin{array}{c}\text { DDM } \\
(\%)\end{array}$ & $\begin{array}{l}\text { DCP } \\
(\%)\end{array}$ & $\begin{array}{c}\text { DEE } \\
(\%)\end{array}$ & $\begin{array}{l}\text { DCF } \\
(\%)\end{array}$ & $\begin{array}{l}\text { Authors } \\
\text { (Country) }\end{array}$ \\
\hline $\mathrm{kg} / 100 \mathrm{~kg})$ & $\begin{array}{l}\text { DCP, Lime stone, Salt, } \\
\text { Lysine, Methionine, } \\
\text { Premix }\end{array}$ & & & & & \\
\hline $\begin{array}{l}\text { Okara }(0 ; 2.375 ; 4.75 ; \\
7.125 ; 9.50 ; 9.50 \mathrm{~kg} / 100 \\
\mathrm{~kg}) \text { Cassava peel meal }(0 ; \\
0.375 ; 0.750 ; 1.125 ; 1.50 \\
1.50 \mathrm{~kg} / 100 \mathrm{~kg})\end{array}$ & $\begin{array}{l}\text { Okara, Cassava peel } \\
\text { meal, Maize, Soybean } \\
\text { meal, GNC, Wheat } \\
\text { offal, BDG, Palm oil, } \\
\text { Fish meal, Bone meal, } \\
\text { Oyster shell, DL } \\
\text { Methionine, Lysine, } \\
\text { Premix, Nacl }\end{array}$ & $\begin{array}{l}72.36- \\
79.79\end{array}$ & $\begin{array}{l}79.04- \\
81.36\end{array}$ & $\begin{array}{l}80.96- \\
84.56\end{array}$ & $\begin{array}{c}46.51- \\
53.64\end{array}$ & $\begin{array}{l}\text { Silas et al., } \\
2014 \\
\text { (Nigéria) }\end{array}$ \\
\hline $\begin{array}{l}\text { Palm kernel meal }(0 ; 10 ; \\
20 ; 30 ; 40 \mathrm{~kg} / 100 \mathrm{~kg}) \\
\text { supplemented enzyme } \\
\text { Hemicell }(0 ; 0.05 \mathrm{~kg} / 100 \\
\mathrm{kg}) \text {, RoxazymeG }(0 ; 0.02 \\
\mathrm{kg} / 100 \mathrm{~kg})\end{array}$ & $\begin{array}{l}\text { Palm kernel meal, } \\
\text { Hemicell, } \\
\text { RoxazymeG, Maize, } \\
\text { Soybean meal, Palm } \\
\text { oil, Bone } \\
\text { meal,Limestone, Salt, } \\
\text { Premix, Methionine, } \\
\text { Lysine, Fish meal }\end{array}$ & $\begin{array}{l}58.43- \\
71.18\end{array}$ & $\begin{array}{c}46.59- \\
60.12\end{array}$ & $\begin{array}{c}49.34- \\
68.05\end{array}$ & $\begin{array}{l}28.31- \\
39.91\end{array}$ & $\begin{array}{c}\text { Aya et al., } 2013 \\
\text { (Nigéria) }\end{array}$ \\
\hline
\end{tabular}

Senna obtusifolia seed meal $(0 ; 20 \mathrm{~kg} / 100 \mathrm{~kg})$ Raw Senna obtusifolia seed meal $(0 ; 20 \mathrm{~kg} / 100$ $\mathrm{kg})$ Boiled Senna obtusifolia seed meal ( 0 ; $20 \mathrm{~kg} / 100 \mathrm{~kg}$ ) Soaked Senna obtusifolia seed meal $(0 ; 20 \mathrm{~kg} / 100 \mathrm{~kg})$ Fermented Senna obtusifolia seed meal ( 0 ;

Senna obtusifolia seed meal (raw, boiled, soaked, fermented), Maize, Maize offal, Soya bean meal, Fishmeal, Groundnut cake, Salt, Bone meal, Methionine, Lysine, Premix

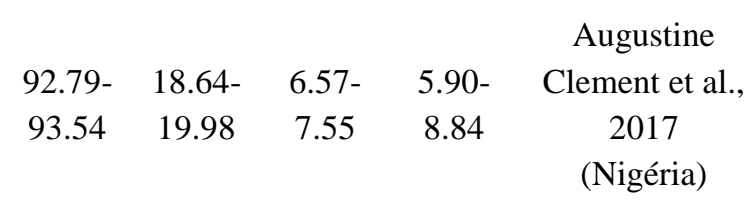

$20 \mathrm{~kg} / 100 \mathrm{~kg} / 100 \mathrm{~kg}$ )

\begin{tabular}{|c|c|c|c|c|c|c|}
\hline $\begin{array}{l}\text { Moringa leaf meal }(0 ; 5 \text {; } \\
10 ; 15 ; 20 \mathrm{~kg} / 100 \mathrm{~kg})\end{array}$ & $\begin{array}{l}\text { Moringa leaf meal, } \\
\text { Maize, Soybean meal, } \\
\text { Groundnut cake, Fish } \\
\text { meal, Wheat offal, } \\
\text { Brewwer Dried grains, } \\
\text { Palm oil, Bone meal, } \\
\text { Oyster shell, Salt, } \\
\text { Premix, Methionine, } \\
\text { Lysine, }\end{array}$ & $\begin{array}{l}58.06- \\
64.44\end{array}$ & ND & $\begin{array}{c}61.48- \\
68.71\end{array}$ & $\begin{array}{l}22.15- \\
23.59\end{array}$ & $\begin{array}{l}\text { Tijani et al., } \\
2016 \\
\text { (Nigéria) }\end{array}$ \\
\hline Maize $(56 \mathrm{~kg} / 100 \mathrm{~kg})$ & $\begin{array}{l}\text { Maize, Wheat bran, } \\
\text { Whole soyabean, Fish } \\
\text { meal, Bone meal, } \\
\text { Premix, Methionine }\end{array}$ & 89.56 & 92.77 & 87.83 & 67.46 & $\begin{array}{l}\text { Kwari et al., } \\
2011 \\
\text { (Nigéria) }\end{array}$ \\
\hline
\end{tabular}




\begin{tabular}{|c|c|c|c|c|c|c|}
\hline $\begin{array}{l}\text { Experimental Feedstuffs } \\
\text { (Rate in the feed) }\end{array}$ & Feed composition & $\begin{array}{c}\text { DDM } \\
(\%)\end{array}$ & $\begin{array}{c}\text { DCP } \\
(\%)\end{array}$ & $\begin{array}{c}\text { DEE } \\
(\%)\end{array}$ & $\begin{array}{r}\text { DCF } \\
(\%)\end{array}$ & $\begin{array}{l}\text { Authors } \\
\text { (Country) }\end{array}$ \\
\hline $\begin{array}{l}\text { Sorghum varieties (56 } \\
\mathrm{kg} / 100 \mathrm{~kg} \text { ) }\end{array}$ & $\begin{array}{l}\text { Wheat bran, Whole } \\
\text { soyabean, Fish meal, } \\
\text { Bone meal, Premix, } \\
\text { Methionine }\end{array}$ & $\begin{array}{l}87.34- \\
88.89\end{array}$ & $\begin{array}{l}87.06- \\
95.87\end{array}$ & $\begin{array}{l}83.65- \\
92.95\end{array}$ & $\begin{array}{c}65.62- \\
68.70\end{array}$ & $\begin{array}{l}\text { Kwari et al., } \\
2011 \\
\text { (Nigéria) }\end{array}$ \\
\hline $\begin{array}{l}\text { Maize bran }(0 ; 10 ; 20 ; 30 \\
\mathrm{kg} / 100 \mathrm{~kg})\end{array}$ & $\begin{array}{l}\text { Maize bran, maize, } \\
\text { Wheat bran, } \\
\text { Cottonseed cake, } \\
\text { Soybean meal, Palm } \\
\text { oil, Lysine, Methionine } \\
\text {, Oyster shell, DCP, } \\
\text { Premix } \\
\text { Salt }\end{array}$ & $\begin{array}{l}75.40- \\
77.28\end{array}$ & ND & ND & ND & $\begin{array}{c}\text { Guédou et al., } \\
2016 \\
\text { (Bénin) }\end{array}$ \\
\hline $\begin{array}{l}\text { Dacryodes edulis Seed } \\
\text { Meal }(0 ; 15 ; 30 ; 45 ; 60 \\
\mathrm{kg} / 100 \mathrm{~kg})\end{array}$ & $\begin{array}{l}\text { Dacryodes edulis Seed } \\
\text { Meal, Maize, Full fat } \\
\text { soybean meal, Blood } \\
\text { meal, Wheat offal, } \\
\text { Fish meal, Bone meal, } \\
\text { Oyster shell, Salt, } \\
\text { Vitamin, Mineral } \\
\text { premix, Methionine }\end{array}$ & $\begin{array}{c}56.30- \\
63\end{array}$ & ND & ND & $\begin{array}{l}40.63- \\
56.28\end{array}$ & $\begin{array}{l}\text { Bratte, } 2010 \\
\text { (Nigéria) }\end{array}$ \\
\hline $\begin{array}{l}\text { Lime juice }(1.00 ; 1.50 ; \\
2.00 ; 2.50 \mathrm{~kg} / 100 \mathrm{~kg})\end{array}$ & $\begin{array}{l}\text { Lime juice, Maize, } \\
\text { Soybean meal, Fish } \\
\text { meal, Palm kernel } \\
\text { cake, Wheat offal, } \\
\text { Bone meal, Salt, } \\
\text { Lysine, Methionine, } \\
\text { Premix }\end{array}$ & $\begin{array}{l}75.05- \\
77.05\end{array}$ & $\begin{array}{c}65.23- \\
78.87\end{array}$ & $\begin{array}{l}74.03- \\
88.23\end{array}$ & $\begin{array}{l}45.05- \\
46.03\end{array}$ & $\begin{array}{l}\text { Ndelekwute } \\
\text { and } \\
\text { Enyenihi, } 2017 \\
\text { (Nigéria) }\end{array}$ \\
\hline $\begin{array}{l}\text { Undecorticated Sunflower } \\
\text { Seed Meal }(0 ; 9 ; 17.8 \text {; } \\
26.4 \mathrm{~kg} / 100 \mathrm{~kg})\end{array}$ & $\begin{array}{l}\text { Undecorticated } \\
\text { Sunflower Seed Meal, } \\
\text { Maize, Fish meal, } \\
\text { Soybean meal, } \\
\text { Sunflower meal, } \\
\text { Groundnut cake, } \\
\text { Wheat offal, Bone } \\
\text { meal, Oyster shell, L- } \\
\text { Lysine-HCl, DL- } \\
\text { Methionine, Premix, } \\
\text { Sodium Chloride }\end{array}$ & $\begin{array}{l}83.30- \\
87.48\end{array}$ & $\begin{array}{l}64.78- \\
68.11\end{array}$ & $\begin{array}{c}60.75- \\
69.61\end{array}$ & $\begin{array}{l}62.94- \\
76.61\end{array}$ & $\begin{array}{c}\text { Faliolu et al., } \\
2015 \\
\text { (Nigéria) }\end{array}$ \\
\hline $\begin{array}{l}\text { Local processed } \\
\text { groundnut cake meal }(0 ; \\
6.50 ; 13.0 ; 19.50 ; 26.0 \\
\mathrm{~kg} / 100 \mathrm{~kg})\end{array}$ & $\begin{array}{l}\text { Local processed } \\
\text { groundnut cake meal, } \\
\text { Maize, Soya beans, } \\
\text { Fish meal, Wheat } \\
\text { offal, Oyster shell, } \\
\text { Bone meal, Premix, } \\
\text { Salt, Lysine, }\end{array}$ & $\begin{array}{l}88.40- \\
91.60\end{array}$ & $\begin{array}{l}76.77- \\
91.67\end{array}$ & $\begin{array}{l}85.87- \\
94.60\end{array}$ & $\begin{array}{l}57.83- \\
83.80\end{array}$ & $\begin{array}{c}\text { Aguihe et al., } \\
2013 \\
\text { (Nigéria) }\end{array}$ \\
\hline
\end{tabular}




\begin{tabular}{|c|c|c|c|c|c|c|}
\hline $\begin{array}{l}\text { Experimental Feedstuffs } \\
\text { (Rate in the feed) }\end{array}$ & Feed composition & $\begin{array}{c}\text { DDM } \\
(\%)\end{array}$ & $\begin{array}{l}\text { DCP } \\
(\%)\end{array}$ & $\begin{array}{c}\text { DEE } \\
(\%)\end{array}$ & $\begin{array}{l}\text { DCF } \\
(\%)\end{array}$ & $\begin{array}{l}\text { Authors } \\
\text { (Country) }\end{array}$ \\
\hline & Methionine & & & & & \\
\hline $\begin{array}{l}\text { Treated Banana Peel Meal } \\
(0 ; 5.64 ; 11.28 ; 16.92 \\
\mathrm{kg} / 100 \mathrm{~kg})\end{array}$ & $\begin{array}{l}\text { Treated Banana Peel } \\
\text { Meal, Maize, Soybean } \\
\text { Meal, Groundnut cake, } \\
\text { Brewers Dry Grain, } \\
\text { Wheal Offal, Palm oil, } \\
\text { Fish Meal, Bone Meal, } \\
\text { Oyster Shell, Lysine, } \\
\text { Methionine, Premix, } \\
\text { Salt }\end{array}$ & $\begin{array}{c}81.63- \\
87.21\end{array}$ & $\begin{array}{c}79.38- \\
87.52\end{array}$ & $\begin{array}{l}90.78- \\
93.39\end{array}$ & $\begin{array}{c}71.09- \\
76.36\end{array}$ & $\begin{array}{l}\text { Fas et al., } 2015 \\
\text { (Nigéria) }\end{array}$ \\
\hline $\begin{array}{l}\text { Fresh bovine } \\
\text { rumen epithelia tissues } \\
\text { scrapings }(0 ; 1.25 ; 2.5 \\
\mathrm{kg} / 100 \mathrm{~kg})\end{array}$ & $\begin{array}{l}\text { Fresh bovine } \\
\text { rumen epithelia tissues } \\
\text { scrapings, Maize, } \\
\text { groundnut Cake } \\
\text { Soybean meal, Palm } \\
\text { kernel Cake, Fish } \\
\text { meal, Wheat offal, } \\
\text { Oyster shell, Bone } \\
\text { meal, Salt, Methionine, } \\
\text { Lysine, Vit./Mineral } \\
\text { Premix }\end{array}$ & $\begin{array}{c}68.82- \\
69.87\end{array}$ & $\begin{array}{l}71.81- \\
81.77\end{array}$ & $\begin{array}{l}67.78- \\
69.43\end{array}$ & $\begin{array}{l}51.43- \\
66.44\end{array}$ & $\begin{array}{l}\text { Salami et al., } \\
2013 \\
\text { (Nigéria) }\end{array}$ \\
\hline $\begin{array}{l}\text { Low energy and high } \\
\text { crude protein, high energy } \\
\text { and low crude protein, } \\
\text { normal energy and normal } \\
\text { crude protein, Low energy } \\
\text { and low crude protein }\end{array}$ & $\begin{array}{l}\text { Maize, Maize offal, } \\
\text { Soybean meal, } \\
\text { Groundnut meal, Fish } \\
\text { meal, Bone meal, } \\
\text { Oyster shell, } \mathrm{NaCl}, \\
\text { Vitamins and minerals } \\
\text { premix, Lysine, } \\
\text { Methionine, Palm oil } \\
\text { sludge }\end{array}$ & $\begin{array}{c}60.3- \\
69.3\end{array}$ & $\begin{array}{c}52.0- \\
62.0\end{array}$ & $\begin{array}{l}60.7- \\
69.1\end{array}$ & $\begin{array}{l}68.5- \\
70.3\end{array}$ & $\begin{array}{l}\text { Dairo et al., } \\
2010 \\
\text { (Nigéria) }\end{array}$ \\
\hline $\begin{array}{l}\text { Fermented Terminalia } \\
\text { catappa whole fruit meal } \\
(0 ; 9 ; 18 ; 27 ; 36 \mathrm{~kg} / 100 \\
\mathrm{kg})\end{array}$ & $\begin{array}{l}\text { Fermented Terminalia } \\
\text { catappa } \text { whole fruit } \\
\text { meal, Maize, Soybean } \\
\text { meal, Wheat offal, } \\
\text { Cassava flour, Fish } \\
\text { meal, Blood meal, } \\
\text { Bone meal, Oyster } \\
\text { shell, DL-Methionine, } \\
\text { Lysine, Vit/min } \\
\text { premix, Salt }\end{array}$ & ND & $\begin{array}{l}62.80- \\
72.10\end{array}$ & $\begin{array}{l}58.60- \\
75.40\end{array}$ & $\begin{array}{l}25.70- \\
42.30\end{array}$ & $\begin{array}{l}\text { Apata, } 2010 \\
\text { (Nigéria) }\end{array}$ \\
\hline $\begin{array}{l}\text { Degraded brewer dried } \\
\text { grains }(0 ; 3 ; 5 ; 7 \mathrm{~kg} / 100 \\
\mathrm{kg})\end{array}$ & $\begin{array}{l}\text { Degraded brewer dried } \\
\text { grains, Maize, Wheat } \\
\text { offal, Groundnut cake, }\end{array}$ & $\begin{array}{l}75.05- \\
80.04\end{array}$ & $\begin{array}{l}73.93- \\
79.73\end{array}$ & $\begin{array}{l}53.82- \\
62.54\end{array}$ & $\begin{array}{l}50.54- \\
57.32\end{array}$ & $\begin{array}{c}\text { Lawal et al., } \\
2016 \\
\text { (Nigéria) }\end{array}$ \\
\hline
\end{tabular}




\begin{tabular}{|c|c|c|c|c|c|c|}
\hline $\begin{array}{l}\text { Experimental Feedstuffs } \\
\text { (Rate in the feed) }\end{array}$ & Feed composition & $\begin{array}{c}\text { DDM } \\
(\%)\end{array}$ & $\begin{array}{l}\text { DCP } \\
(\%)\end{array}$ & $\begin{array}{l}\text { DEE } \\
(\%)\end{array}$ & $\begin{array}{r}\text { DCF } \\
(\%)\end{array}$ & $\begin{array}{l}\text { Authors } \\
\text { (Country) }\end{array}$ \\
\hline & $\begin{array}{l}\text { Soya bean meal, Fish } \\
\text { meal, Bone meal, } \\
\text { Oyster shell, Premix, } \\
\text { Salt, Lysine, } \\
\text { Methionine }\end{array}$ & & & & & \\
\hline $\begin{array}{l}\text { Undegraded brewer dried } \\
\text { grains }(0 ; 7 \mathrm{~kg} / 100 \mathrm{~kg})\end{array}$ & $\begin{array}{l}\text { Undegraded brewer } \\
\text { dried grains, Maize, } \\
\text { Wheat offal, } \\
\text { Groundnut cake, Soya } \\
\text { bean meal, Fish meal, } \\
\text { Bone meal, Oyster } \\
\text { shell, Premix, Salt, } \\
\text { Lysine, Methionine }\end{array}$ & $\begin{array}{l}70.90- \\
71.37\end{array}$ & $\begin{array}{l}68.88- \\
69.70\end{array}$ & $\begin{array}{l}46.24- \\
49.30\end{array}$ & $\begin{array}{l}34.53- \\
35.46\end{array}$ & $\begin{array}{l}\text { Lawal et al., } \\
2016 \\
\text { (Nigéria) }\end{array}$ \\
\hline $\begin{array}{l}\text { Urea fermented brewer's } \\
\text { dried grains }(0 ; 8.32 \\
16.70 ; 25.00 ; 33.5 \mathrm{~kg} / 100 \\
\mathrm{~kg})\end{array}$ & $\begin{array}{l}\text { Urea fermented } \\
\text { brewer's dried grains, } \\
\text { Maize (Yellow), } \\
\text { Groundnut cake, Fish } \\
\text { meal, Blood meal, } \\
\text { Oyster shell, Bone } \\
\text { meal, Palm oil, } \\
\text { Premix, Methionine, } \\
\text { Salt }\end{array}$ & $\begin{array}{l}60.19- \\
81.73\end{array}$ & $\begin{array}{l}13.27- \\
20.05\end{array}$ & ND & ND & $\begin{array}{l}\text { Isikwenu, } 2008 \\
\text { (Nigéria) }\end{array}$ \\
\hline $\begin{array}{l}\text { Sweet orange peel meal } \\
(0 ; 4.90 ; 9.80 ; 14.70 ; \\
19.60 ; 24.50 \mathrm{~kg} / 100 \mathrm{~kg})\end{array}$ & $\begin{array}{l}\text { Sweet orange peel } \\
\text { meal, Maize, Soybean } \\
\text { meal, Brewers dried } \\
\text { grain, Blood meal, } \\
\text { Bone meal, Common } \\
\text { salt, Methionine, } \\
\text { Lysine, } \\
\text { Vitamin/mineral } \\
\text { premix }\end{array}$ & ND & $\begin{array}{l}60.23- \\
64.48\end{array}$ & $\begin{array}{l}62.69- \\
66.88\end{array}$ & $\begin{array}{l}57.49- \\
62.83\end{array}$ & $\begin{array}{c}\text { Oluremi et al. } \\
2017 \\
\text { (Nigéria) }\end{array}$ \\
\hline $\begin{array}{l}\text { Sorghum varieties ( } 45 \\
\mathrm{~kg} / 100 \mathrm{~kg} \text { ) supplemented } \\
\text { with } 500 \mathrm{FTU} \text { microbial } \\
\text { phytase and } 1600 \mathrm{BXU} \\
\text { xylanase }\end{array}$ & $\begin{array}{l}\text { Sorghum varieties, } \\
\text { Soyabean, L-lysine, } \\
\text { DL-Methionine, } \\
\text { Vitamin-mineral } \\
\text { premix, Limestone, } \\
\text { Salt, Monocalcium } \\
\text { phosphate, Sodium } \\
\text { bicarbonate, Oil- } \\
\text { sunflower, Celite }\end{array}$ & ND & $\begin{array}{c}61.0- \\
66.0\end{array}$ & $\begin{array}{c}66.0- \\
77.0\end{array}$ & ND & $\begin{array}{c}\text { Mabelebele et } \\
\text { al., } 2017 \\
\text { (South Africa) }\end{array}$ \\
\hline Corn $(91.1 \mathrm{~kg} / 100 \mathrm{~kg})$ & $\begin{array}{l}\text { Soy oil, Salt, } \\
\text { Limestone, } \\
\text { Monocalcium } \\
\text { phosphate, Chromic } \\
\text { oxide premix, Choline }\end{array}$ & ND & 63.3 & ND & ND & $\begin{array}{c}\text { Iyayi } \\
\text { and Adeola, } \\
2014 \\
\text { (Nigéria) }\end{array}$ \\
\hline
\end{tabular}




\begin{tabular}{|c|c|c|c|c|c|c|}
\hline $\begin{array}{l}\text { Experimental Feedstuffs } \\
\text { (Rate in the feed) }\end{array}$ & Feed composition & $\begin{array}{c}\text { DDM } \\
(\%)\end{array}$ & $\begin{array}{l}\text { DCP } \\
(\%)\end{array}$ & $\begin{array}{l}\text { DEE } \\
(\%)\end{array}$ & $\begin{array}{l}\text { DCF } \\
(\%)\end{array}$ & $\begin{array}{c}\text { Authors } \\
\text { (Country) }\end{array}$ \\
\hline & $\begin{array}{l}\text { chloride, Vit-min } \\
\text { premix }\end{array}$ & & & & & \\
\hline Sorghum $(85.0 \mathrm{~kg} / 100 \mathrm{~kg})$ & $\begin{array}{l}\text { Soy oil, Salt, } \\
\text { Limestone, Dextrose, } \\
\text { Monocalcium } \\
\text { phosphate, Chromic } \\
\text { oxide premix, Choline } \\
\text { chloride, Vit-min } \\
\text { premix }\end{array}$ & ND & 66.9 & ND & ND & $\begin{array}{c}\text { Iyayi } \\
\text { and Adeola, } \\
2014 \\
\text { (Nigéria) }\end{array}$ \\
\hline $\begin{array}{l}\text { Full fat soybean (55.6 } \\
\mathrm{kg} / 100 \mathrm{~kg})\end{array}$ & $\begin{array}{l}\text { Soy oil, Salt, } \\
\text { Limestone, Dextrose, } \\
\text { Monocalcium } \\
\text { phosphate, Chromic } \\
\text { oxide premix, Choline } \\
\text { chloride, Vit-min } \\
\text { premix }\end{array}$ & ND & 78.8 & ND & ND & $\begin{array}{c}\text { Iyayi } \\
\text { and Adeola, } \\
2014 \\
\text { (Nigéria) }\end{array}$ \\
\hline $\begin{array}{l}\text { Fish meal }(32.6 \mathrm{~kg} / 100 \\
\mathrm{kg})\end{array}$ & $\begin{array}{l}\text { Soy oil, Salt, } \\
\text { Limestone, Dextrose, } \\
\text { Monocalcium } \\
\text { phosphate, Chromic } \\
\text { oxide premix, Choline } \\
\text { chloride, Vit-min } \\
\text { premix }\end{array}$ & ND & 82.1 & ND & ND & $\begin{array}{c}\text { Iyayi } \\
\text { and Adeola } \\
2014 \\
\text { (Nigéria) }\end{array}$ \\
\hline $\begin{array}{l}\text { Peanut flour }(31.1 \mathrm{~kg} / 100 \\
\mathrm{kg})\end{array}$ & $\begin{array}{l}\text { Soy oil, Salt, } \\
\text { Limestone, Dextrose, } \\
\text { Monocalcium } \\
\text { phosphate, Chromic } \\
\text { oxide premix, Choline } \\
\text { chloride, Vit-min } \\
\text { premix }\end{array}$ & ND & 81.7 & ND & ND & $\begin{array}{c}\text { Iyayi } \\
\text { and Adeola } \\
2014 \\
\text { (Nigéria) }\end{array}$ \\
\hline $\begin{array}{l}\text { Wheat bran }(48.1 \mathrm{~kg} / 100 \\
\mathrm{kg})\end{array}$ & $\begin{array}{l}\text { Soy oil, Salt, } \\
\text { Limestone, Dextrose, } \\
\text { Monocalcium } \\
\text { phosphate, Chromic } \\
\text { oxide premix, Choline } \\
\text { chloride, Vit-min } \\
\text { premix }\end{array}$ & ND & 38.1 & ND & ND & $\begin{array}{c}\text { Iyayi } \\
\text { and Adeola, } \\
2014 \\
\text { (Nigéria) }\end{array}$ \\
\hline
\end{tabular}

DDM= Dry Matter Digestibility; DCP= Digestibility Crude Protein; DEE= Ether Extract Digestibility; DCF= Crude Fiber Digestibility; ND= Not Determined 


\section{Conclusion}

A total of twenty-five (25) scientific articles were used to provide information on balanced feed digestibility in broiler chicken, in Africa. The data demonstrate that the compound feeds delivered to chicken are often digestible. This inventory will be useful to assess the digestibility of each of concentrated feedstuffs in broiler chicken to develop a table of their nutritional composition in Benin.

\section{COMPETING INTERESTS}

The authors declare that they have no competing interests.

\section{AUTHORS' CONTRIBUTIONS}

GSTA collected scientific papers on feed digestibility in broiler chicken published from 2008 to 2018 in Africa. After compiling and analyzing informations collected, she wrote the paper draft. Après avoir compilé et analysé les informations collectées, elle a rédigé le projet d'article. FMH, CAAMC and GAM read and corrected the paper draft before submission.

\section{ACKNOWLEDGMENTS}

The authors thank all those who contributed to the realization of this review, in particular the University of Abomey-Calavi (UAC) and the National Institute of Agricultural Research of Benin (INRAB).

\section{REFERENCES}

Adoum IY. 2007. Qualité nutritionnelle des provendes à base d'amande de coton chez les poulets de chair: étude comparative des variétés «glandless» «glanded». Advanced degree, EISMV, Sénégal, p. 44.

Aguihe PC, Fatokun BO, Samuel AS, Aguihe KU EO, Osaguona PO. 2013. Growth Performance and Nutrient Digestibility of Broiler Chickens Fed Diet with Graded Levels of Local Processed Groundnut Cake Meal as Replacement for Soybean Meal. Inter J Agri Biosci, 2(5): 256-259. www.ijagbio.com.

AOAC. 1991. Official Methods of Analysis $\left(15^{\text {th }}\right.$ Edn). Association of Official Agricultural Chemists: Washington, DC, USA.

AOAC. 2000. Official Methods of Analysis $\left(18^{\text {th }}\right.$ Edn). Association of Official Agricultural Chemists: Washington, DC, USA.

Apata DF. 2010. Effect of Terminalia catappa Fruit Meal Fermented by Aspergillus niger as Replacement of Maize on Growth Performance, Nutrient Digestibility, and Serum Biochemical Profile of Broiler Chickens. Biotechnology Research International, 2011: $907546, \quad 6 p$. DOI:10.4061/2011/907546

Augustine C, Dankasa IK, Uchei IJ, Bala AS, Siaka DS. 2017. Nutrient digestibility and growth performance of broiler chickens fed processed tropical sicklepod (Senna obtusifolia (1.)) Seed meal based-diets. Journal of Agricultural Sciences, 62(4): 371-384. DOI: https://dx.doi.org/10.2298/JAS1704371C

Archimède H, Bastianelli D, Boval M, Tran G, Sauvant D. 2011. Ressources tropicales: disponibilité et valeur alimentaire. INRA Prod. Anim., 24(1): 23-40.

Aya VE, Ayanwale BA, Ijaiya AT, Aremu A. 2013. Performance and nutrient digestibility in broiler chicks as influenced by multienzyme addition to starter diets containing palm kernel meal. Biotechnology in Animal Husbandry, 29(1): 93-104. DOI: 10.2298/BAH1301093A

Bratte L, Mmereole FUC, Akpodiete OJ, Omeje SI. 2010. The Nutrient Composition of Seeds of the African Pear (Dacryodes edulis) and its Implications for Non-Ruminant Nutrition. Pakistan Journal of Nutrition, 9: $255-258$. 
Bourdillon A, Carre B, Conan L, Francesch M, Fuentes M, Huyghebaert G, Janssen WMMA, Leclercq B, Lessire M, McNab J, Rigoni M, Wiseman J. 1990. European reference method of in vivo determination of metabolisable energy in poultry: reproducibility, effect of age, comparison with predicted values. Brit. Poult. Sci., 31: 567-576. DOI: 10.1080/00071669008417288

Dairo FAS, Adesehinwa AOK, Oluwasola TA, Oluyemi JA. 2010. High and low dietary energy and protein levels for broiler chickens. African Journal of Agricultural Research, 5(15): 20302038. DOI: $10.5897 / A J A R 10.254$

Deiltel L, Créchet C, Fornier E, Leborgne MC, Montméas L. 2012. Nutrition et Alimentation des Animaux d'Elevage (Tome I, 3ème édition). Ed. Educagri: Dijon.

Dimi SGR. 1990. Étude des possibilités d'incorporation de graines de légumineuses dans l'alimentation de poulets de chair : cas de l'arachide et du niébé. Agricultural Engineer's thesis, ENSA (Thiès), Sénégal, p. 92.

Faliolu AO, Oduguwa OO, Jegede AV, Tukura CC, Olarotimi ID, Teniola AA, Alabi JO. 2015. Assessment of enzyme supplementation on growth performance and apparent nutrient digestibility in diets containing undecorticated sunflower seed meal in layer chicks. Poultry Science, 94: 1917-1922. DOI: http://dx.doi.org/10.3382/ps/pev136

Fas A, Adeyemi OA, Oluwole OB, Oladunmoye OO, Ayo-Ajasa OY. 2015. Effects of Treated Banana Peel Meal on the Feed Efficiency, Digestibility and Cost Effectiveness of Broiler Chickens Diet. J Vet Sci Anim Husb, 3(1): 101.

Guédou MSE, Houndonougbo MF, Chrysostome CAAM, Mensah GA. 2016. Performances zootechniques et économiques de coquelets nourris avec des aliments à base de quatre variétés de grains de maïs au Bénin. Rev. Cames-
Sciences de la Vie, de la Terre et Agronomie, 4(1): 44-50.

Guermah H. 2012. Nutrition du lapin : Etude de sources alimentaires alternatives. PhD thesis, Tizi-Ouzou University Mouloud Mammeri, p.122.

Guindjoumbi S. 2007. Cuniculture périurbaine dans les Niayes : Situation actuelle et perspectives de développement. $\mathrm{PhD}$ thesis in Veterinary Medecine, University of Dakar, p. 117.

Igwebuike JU, Medugu CI, Kwari ID, Augustine C, Peter J, Bashir IM. 2017. Growth Performance, Nutrient Digestibility and Carcass Characteristics of Broiler Chickens Fed Moringa (Moringa oleifera) and Baobab (Adansonia digitata) Leaf Powders to Replace Synthetic Premix in North-East - Nigeria. Journal of Scientific and Engineering Research, 4(12): 10-16.

INRA. 1989. L'alimentation des animaux monogastriques: porc; lapin; volailles. (2nd edn).INRA: Paris.

Isikwenu JO, Omeje SI, Okagbare G, Akpodiete OJ. 2008. Effect of replacing groundnut cake with urea fermented brewer's dried grains in broiler chicks' diets. Animal Research International, 5(1): 795-800.

Iyayi EA, Adeola O. 2014. Standardized ileal amino acid digestibility of feedstuffs in broiler chickens. Europ. Poult. Sci., 78 : 2014. DOI: $10.1399 /$ eps.2014.54

Kouzoukende TH. 2000. Interrelation hygiène et performances des poulets de chair en aviculture moderne dans la région de Dakar. $\mathrm{PhD}$ thesis in Veterinary Medecine, University of Dakar, p. 120.

Kpodékon TM, Youssao AKI, Koutinhouin BG, Missohou A, Fayomi J, Fagbohou A, Djago Y. 2009. Comparaison des performances de croissance de lapereaux en engraissement nourris par un aliment à base de tourteau de tournesol, soit sous forme farineuse soit sous forme granulée. Livestock Research For Rural Development, 12(21) 2009. 
Kwari ID, Saleh B, Diarra SS, Mkighir T, Umanah MJ. 2011. Nutrient digestibility and carcass characteristics of broiler chickens fed different cultivars of sorghum replacing maize in the semiarid zone of Nigeria. Research Opinions in Animal \& Veterinary Sciences, 1(9): 578-581.

Lawal TE, Ademola SG, Owoseni AA, Atobatele OE, Asuelimen PO. 2016. Aspergillus flavus Degraded Brewer Dried Grains for Broiler Chicken Diet: Performance and Nutrient Digestibility Parameters. Journal of Advances in Biology \& Biotechnology, 11(1): 1-8. DOI: $10.9734 / \mathrm{JABB} / 2017 / 286811 \mathrm{y}$

Mabelebele M, Gous RM, Siwela M, O'Neil HVM, Iji PA. 2017. Performance of broiler chickens fed South African sorghum-based diets with xylanase. South African Journal of Animal Science, 2(5): $47 . \quad$ DOI: 10.4314/sajas.v47i5.11

Mahoungou-mouéllé NM. 1996. Etude de la valeur alimentaire des principales céréales disponibles au Sénégal (mil, maïs, sorgho) chez le poulet de race locale (Gallus sp) et la pintade (Numida meleagris). Agricultural Engineer's thesis, ENSA (Thiès), Sénégal, p. 92.

N'gom S. 2004. Ebauche d'un referentiel sur la composition chimique et valeur nutritive des matieres premieres utilisables en alimentation des volailles au senegal. Thèse de doctorat de troisième cycle de chimie et biochimie des produits naturels. $\mathrm{PhD}$ thesis, University of Cheick Anta Diop, Dakar, p. 158 .

Ndelekwute EK, Enyenihi GE. 2017. Lime Juice as a Source of Organic Acids for Growth and Apparent Nutrient Digestibility of Broiler Chickens. J Vet Med Surg, 1: 1.

Ndelekwute EK, Enyenihi GE, Unah UL, Madu HC. 2016. Dietary effects of different organic acids on growth and nutrient of different organic acids on growth and nutrient digestibility of broiler of different organic acids on growth and nutrient. Bang. J. Anim. Sci., 45(2): $\quad 10-17 . \quad$ DOI: 10.3329/bjas.v45i2.29802

Obun CO. 2008. Performance, digestibility and carcass and organ weights of finisher broiler chicks fed graded levels of fermented locust bean (Parkia biglobosa) seed meal. Asian Journal of Poultry Science, 2(1): 17-23.

Oluremi OIA, Ahile AA, Jande TF. 2017. Effect of Feeding Graded Levels of Fermented Sweet Orange (Citrus Sinensis) Fruit Peel Meal on the Growth and Nutrient Digestibility of Broiler Chicken. International Journal of Environment, Agriculture and Biotechnology, 2(6): 2017. DOI: http://dx.doi.org/10.22161/ijeab/2.6.44

Raji MO, Ogunjimi JT, Ogunbode AA, Ogungbenro SD, Osuolale SO, Ajani MO. 2015. Performance characteristics and apparent nutrient digestibility of broiler finisher fed African yam bean cake as partial replacement for soybean meal. Arch. Zootec., 64(246): 175-178.

Salami SA, Ali A, Babatunde OT. 2013. Effect of Substitution of Bovine Rumen Epithelial Tissue Scrapings for Fishmeal on the Growth Performance, Nutrient Utilization and Carcass Characteristics of Broiler Chickens. Animal Production 15(1): 15- 23.

Sauvant D. 2004. Principes généraux de l'alimentation animale. INRA: ParisGrignon, p. 147.

Silas AFA, Oluwole OB, Elemo GN, Ajayi AO, Imade A, Sarumi BB, Onyibe J, Mayaki OM, Odediran F, Ebun KK, Egbai HC, Ogunji AO, Asieba G, Erukainure OL, Adeyemi OA. 2014. Growth Performance, Nutrient Digestibility and Haematology of Broiler Chickens Fed Varying Levels of Okara and Cassava Peel Meal. International Journal of Molecular Veterinary 
Research, 4(2): 2014. DOI: 10.5376/ijmvr.2014.04.0002.

Sobayo RA, Oso AO, Adeyemi OA, Fafiolu AO, Jegede AV, Idowu OMO, Dairo OU, Iyerimah RB, Ayoola OA, Awosanya RA. 2012. Changes in growth, digestibility and gut anatomy by broilers fed diets containing ethanoltreated castor oil seed (Ricinus communis L.) meal. Revista Científica UDO Agrícola 12(3): 660-667.

Rivière R. 1991. Manuel d'alimentation des ruminants domestiques en milieu tropical. Paris, France : la documentation française, $529 \mathrm{p}$.

Tijani LA, Akanji AM, Agbalaya K, Onigemo M. 2016. Effects of Moringa oleifera Leaf Meal on Performance, Nutrient Digestibility and Carcass Quality of Broiler Chickens. Applied Tropical Agriculture, 21(1): 46-53.
Toleba SS, Mensah GA, Zougou CGT, Codjo B, Kpéra GN, Pomalégni SCB, 2007. Inventaire des ingrédients alimentaires simples et composés utilisés pour nourrir l'aulacode d'élevage au sud et au centre du Bénin. Bulletin de la Recherche Agronomique du Bénin, 57.

Traore EH, Bebay EC. 2006. Première évaluation de la structure et de l'importance du secteur avicole commercial et familial en Afrique de l'Ouest. FAO, Senegal report, p. 50.

Youssef AA, El-Tahawy WS, El-Hamid EA, Nizza A, Bovera F, Al-Harthi MA, ElKelway MI. 2014. Effect of feed form, pellet diameter and enzymes supplementation on growth performance and nutrient digestibility of broiler during days 21-37 of age. Archiv Tierzucht, 57(34): 1-11. DOI: 10.7482/0003-9438-57-034. 